\title{
GASTROCNEMIUS-PLASTY FOR PRIMARY CLOSURE OF COMPOUND INJURIES OF THE KNEE
}

\author{
Bent Barfod and Michael Pers, Denmark \\ From the Surgical Department II, Arhus County Hospital, Arhus, and \\ the Department of Plastic Surgery, Rigshospitalet, Copenhagen
}

Free skin grafting has become recognised as a valuable part of the primary treatment of traumatic skin defects. However, free skin grafts can be used only when the base of the wound is vascularised. Fascia and muscle are ideal recipient sites whereas covering of tendons, bones and open joints needs pedicle flaps carrying their own blood supply.

When extensive skin loss has occurred around an open joint, it is not possible to close the joint with local skin plastic procedures. It is therefore usually necessary in such cases to employ skin flaps from neighbouring regions-for instance, abdominal flaps for the upper limbs or cross-leg flaps for the lower limbs.

Extensive lesions of this type are seen in the lower limbs when an individual is dragged along the road surface by a motor vehicle. In two such cases, we have used a local muscle flap to cover the gaping knee instead of a flap from the opposite leg.

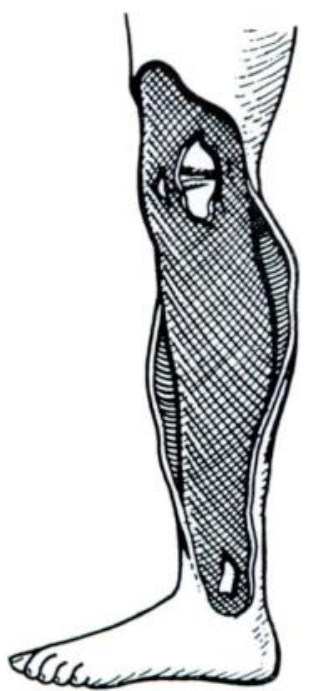

Fig. 1

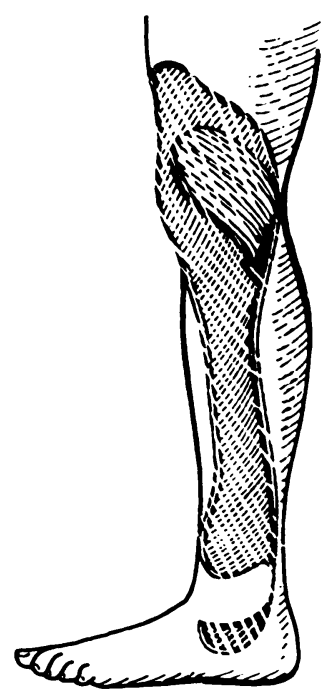

FIG. 2

Case 1. Figure 1-Erosion wound with massive skin loss and gaping joint. Figure 2-Primary closure of the open joint with muscle flap and free skin grafting to the entire defect. (After Pers 1967.)

Treatment-The wound surfaces were cleaned by washing with 3 per cent hydrogen peroxide and irrigated with large quantities of saline. The edges of the loose flap were trimmed until fresh bleeding occurred. The exposed lateral malleolus was covered by displacement of a pedunculated skin flap based anteriorly.

The eroded condyles and the gaping knee were covered with the lateral belly of the gastrocnemius muscle, the muscle being detached from its lower insertion and mobilised so that it could be turned laterally, forwards and upwards and laid over the defect with its original 


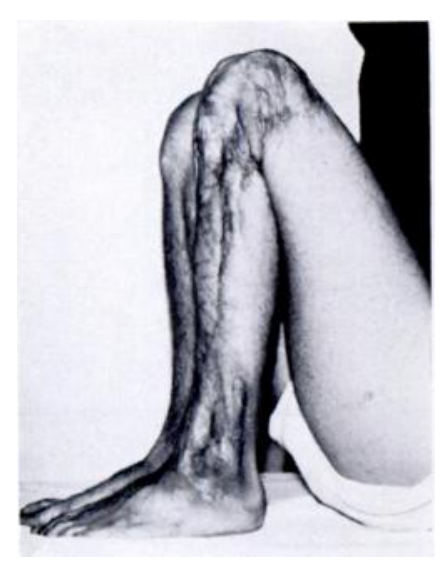

FIG. 3

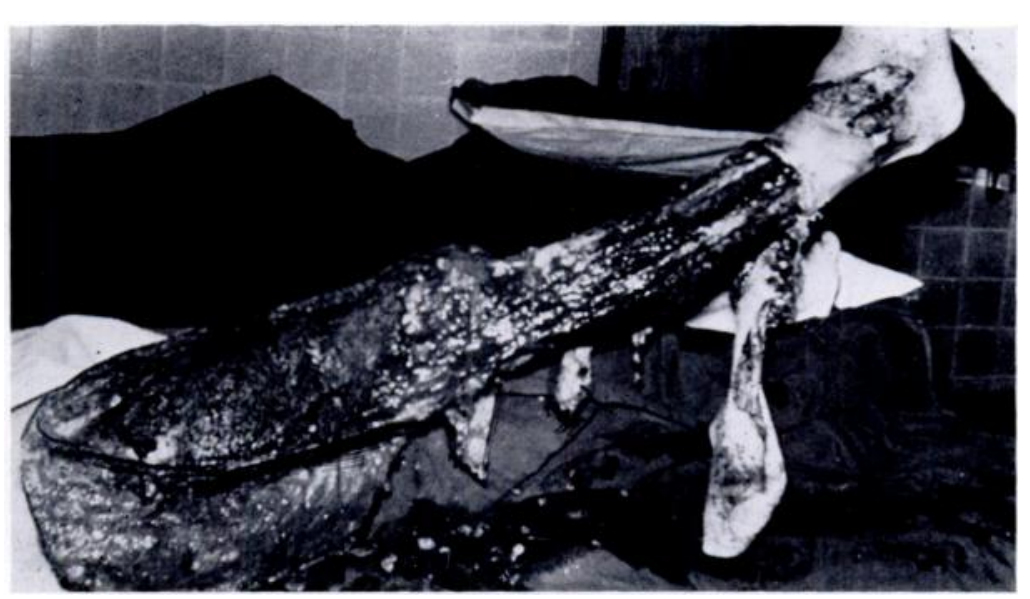

FIG. 4

Figure 3-Case 1. The result eleven years after the primary gastrocnemius plastic procedure on the open knee. Figure 4-Case 2. The right lower limb immediately after injury,

outer surface towards the joint cavity (Fig. 2). The muscle was secured by interrupted catgut sutures and covered together with the remaining skin defect by a moderately thick free skin graft from the right leg. The wounds were not drained.

Progress-The graft was inspected on the seventh day after operation and was found to have taken in its greater part, also over the muscle flap. No bacterial growth was obtained on culture from the wound. From the twelfth day the wound was treated without dressings. Some pustules required opening, and culture yielded staphylococcus aureus. There was no evidence of infection of the joint. Active movements were begun three weeks after the operation. After twelve weeks all the wounds had healed, there was good power in the quadriceps and the knee could be flexed from 0 to 80 degrees: slight lateral instability remained.

When reviewed eleven years after the original injury the patient stated that he had almost normal function of the knee. He was able to ride, swim and play Judo. There was a full range of knee movements and only slight lateral instability (Fig. 3).

Case 2-In November 1967 a thirteen-year-old girl was hit by a bus. The back wheel went over her and she was dragged along the road. Massive avulsion of skin had occurred from the entire right lower limb which was contaminated with road dirt over the muscles and in the intermuscular spaces (Fig. 4). The popliteal artery and vein were intact but traversed an open cavity filled with dirt. The right knee was open on the outer side. The joint capsule, the ilio-tibial tract and part of the bony surfaces on the tibia, the edge of the tibia, the edge of the patella and the fibula had been scraped off. There was gravel in the joint cavity and embedded in the cartilage.

Treatment-The large skin flaps which were attached distally were detached and the least damaged of them were prepared as free whole skin grafts. The fascia and the damaged muscles were detached particularly from the vastas lateralis muscle. The eroded bony surfaces around the knee were smoothed with a chisel and the joint inspected. The earth-filled scratches in the femoral condyles were planed so that the cartilaginous surfaces were clean.

After this a large wound remained in which the muscles were viable and the joint was gaping.

A flap was prepared from the lateral belly of the gastrocnemius, the muscle being detached from the insertion into the calcaneal tendon. This was swung up so that it covered the common peroneal nerve and formed a viable covering over the open joint where it was sewn to the lateral part of the patella and the distal part of the quadriceps tendon (Figs. 5 and 6). 
As the wound involved the entire circumference of the leg, the limb was suspended in a Hoffmann apparatus which was fixed to the tibia. The apparently viable skin flap with proximal attachment on the lateral aspect of the femur was sutured in place.

Skin grafts prepared in advance were applied over the viable femoral muscles: the rest was covered with split-skin grafts.

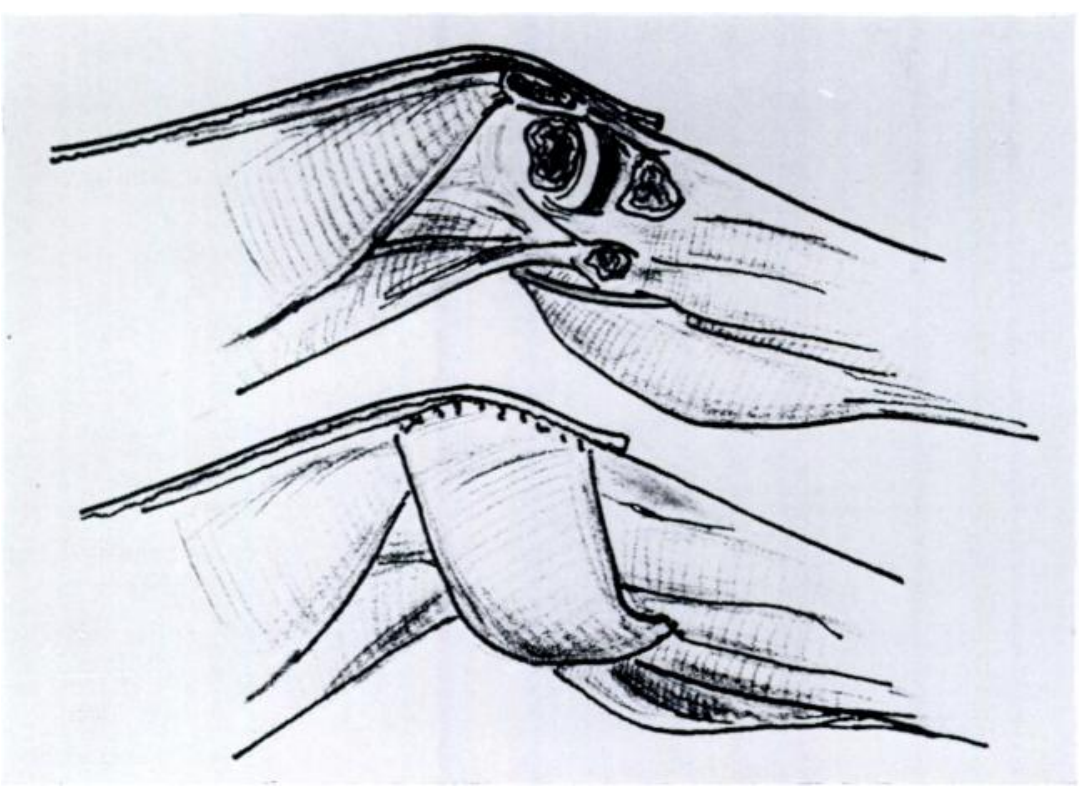

Fig. 5

Case 2-A diagram of the gastrocnemius plastic procedure.

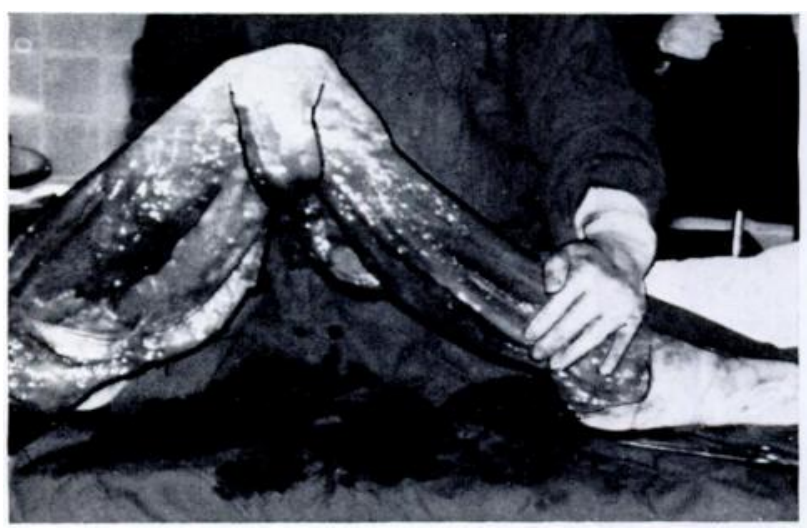

Fig. 6

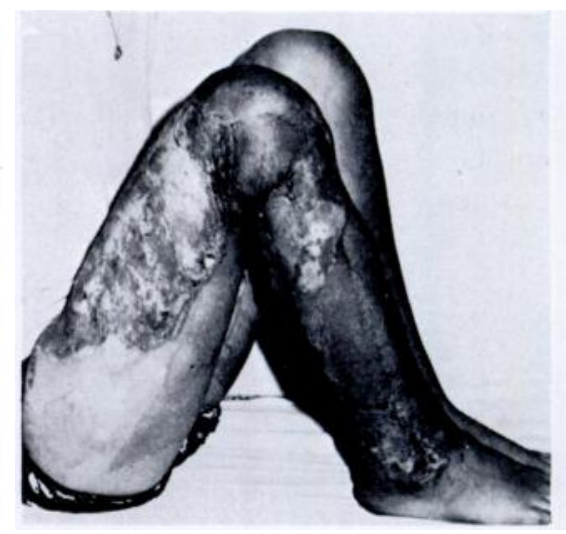

Fig. 7

Case 2. Figure 6-Plastic procedure with the lateral belly of gastrocnemius completed. Figure 7-The legs ten months after injury.

Progress-No post-operative reactions occurred. When the dressing was changed twelve days after the accident, the proximally attached skin flap which had been sutured back to the thigh was found to be necrotic and regrafting was necessary. Twenty days after the accident the skin grafts were found to have taken practically everywhere, and active movements of the knee joint were begun.

A month after the accident the Hoffmann apparatus was discarded. The donor sites were not healed and were treated by irrigation with hypochlorite. Some tension was present over 
the foot because of keloid. Weight-bearing and energetic training were begun and the patient was discharged home three months after the accident.

Three months later the knee could be flexed from 0 to 120 degrees. When the knee was flexed, lateral rotation was slightly increased. The patient had no pain; she could run and cycle normally and play volleyball. Ten months after the accident the right knee could be flexed from 0 to 145 degrees (Fig. 7). Slight lateral mobility was present in the flexed position. The grafted areas remained stable.

\section{DISCUSSION}

A century ago the mortality from an open lesion of a large joint was 70 per cent and it was seldom possible to maintain the function of a knee by conservative treatment. Since the first world war primary closure has been the usual method of choice when circumstances permit (Zeis 1960). Although an adherent of the principle of primary closure of traumatic defects, Brown (1965) advised against emergency cross-leg plastic operations on account of the technical difficulties involved. These are undoubtedly considerable when extensive free skin grafts are required for the damaged limb. Hueston and Gunter (1967) reported good results from the use of primary cross-leg plastic operations for lesions of the calf and foot, but the knee joint was not damaged in any of their cases.

With the gastrocnemius plastic technique described here, it is easy and safe to transform a complicated problem into the more routine task of covering an extensive vascularised wound surface with skin grafts. With this technique it should be possible to close a knee in almost every instance if treatment is instituted within six to eight hours after injury.

Hoffmann's apparatus, which was used in one of these two cases for suspension and immobilisation, is not essential for treatment but is undoubtedly helpful also for cross-leg plastic procedures as described by Eriksson, Eriksson and Nylén (1966). The primary closure of the joint is considered to have been of decisive importance.

\section{SUMMARY}

Two cases of laterally open knee joints with surrounding skin defects are reported. The joints were closed by muscle flaps fashioned from the lateral belly of the gastrocnemius, which was detached distally and folded upwards and forwards to cover the defect. The transposed muscle and the remainder of the wound were covered by free skin grafts. Results were satisfactory.

\section{REFERENCES}

Brown, R. F. (1965): The Management of Traumatic Tissue Loss in the Lower Limb, Especially when Complicated by Skeletal Injury. British Journal of Plastic Surgery, 18, 26.

Eriksson, F., Eriksson, G., and Nylén, B. (1966): Open Fractures: A New Fixation Method in Cross-leg Cases (Arm-Leg). Plastic and Reconstructive Surgery, 38, 410.

Hueston, J. T., and Gunter, G. S. (1967): Primary Cross-Leg Flaps. Plastic and Reconstructive Surgery, 40, 58. PERS, M. (1967): En introduktion til plastikkirurgien. København: Forlaget for faglitteratur.

ZEIS, M. (1960): Fortschritte in der Behandlung der offenen Kniegelenkverletzungen. Zentralblatt für Chirurgie, $85,1202$. 